\title{
A New Demand Modeling for Prolonging the Product Life Cycle based on Triz Theory
}

\author{
Jianqiu Mao \\ University of Jinan \\ Jinan, China \\ Me_maojq@ujn.edu.com \\ Changqing Gao \\ University of Jinan \\ Jinan, China \\ Me_gaocq@ujn.edu.com
}

\author{
Bing Lv \\ University of Jinan \\ Jinan, China \\ Me_lvb@ujn.edu.com
}

\begin{abstract}
In this paper, based on the existing approach and interpolation techniques, we propose a new method to prolong the life-cycle of products with upper level multifunction design. First, the multi-functions of most products are designed for solving the demands of user at the same period. However, these functions are seldom used except the main function. As a result, the original products lifecycle was ended earlier than it is designed. Finally, we solve this problem by using TRIZ innovation theory to obtain the optimal solution set of the original problem.
\end{abstract}

Keywords: Demand modeling;product life-cycle;mutifunction; Continuity; design

\section{INTRODUCTION}

The paper is organized as follows. In the next section, we propose the model that we research in this paper, and some definitions and assumptions are given. In Section 2, we discuss the shortages of the existing approach of multifunction. In these scenarios, successfully managing forecasts and inventories requires advanced demand and inventory modeling technologies in order to reliably support high service levels.[1] Section 3, we solve the existing problem by using TRIZ innovation theory to obtain the optimal solution set of the original problem. In Section 4, a design case was shown to illustrate the efficiency of the new method. Finally, we conclude our paper in section 5 .

\section{THE EXISTING APPROACH OF DEMAND MODELING}

A function model, similar with the activity model or process model, is a graphical representation of an enterprise's function within a defined scope . [2]

In contrast to traditional methods, the new demand modeling methods sense the expected impact of different decisions, understand the distinction between sales channels, anticipate the impact of the decisions, and create a unified view of the demand.

One of the most important methods is modular design; this method emphasizes the products that have different modular functions at the same time. And generate different products through the selection and combination of different function modules. This method of muti-function emphasizes the muti-function at the same time. There are three fundamental advantages of this method. First is, it can provide a clearly clue for product specification. Second, it can diminish the cost of production. Finally, it can reduce the production manufacturing cycle.

These advantages improve both the product quality and the update of products. And it was convenient both the repairing and recycling. But dose the users use all of the functions at the same time? How frequently dose they use the different functions?

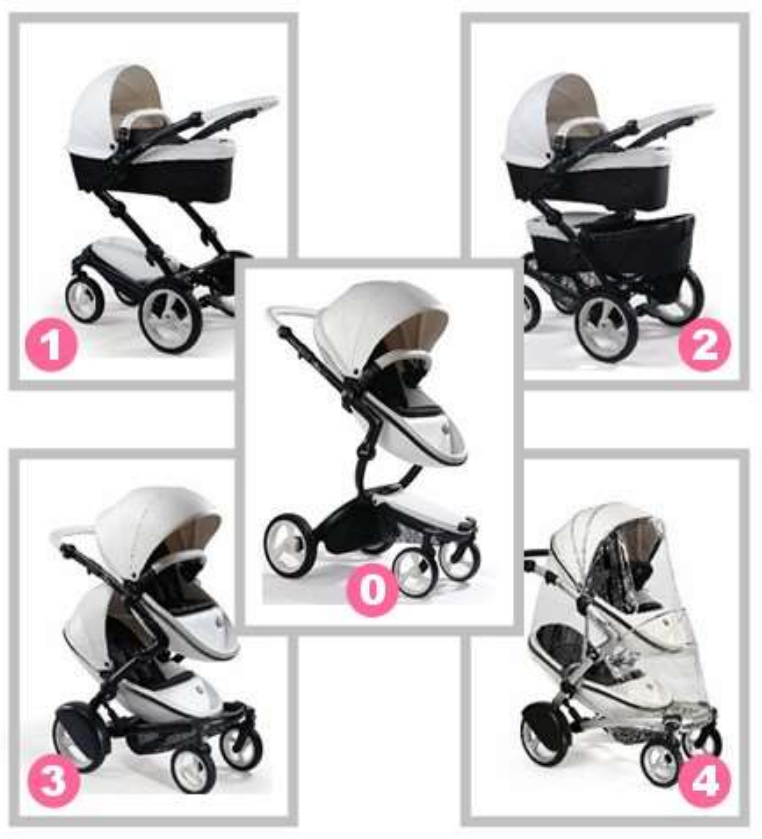

Figurre 1 the muti-function at the same time of MIMA Baby carriage

0. Seat (core functions)

1.lying (expanded function)

2.Lying+Storage (expanded function)

3.seat for twins (expanded function)

4.rainproof (expanded function) 
The product in Figure 1 is a famous baby carriage brand of Holland, the brand heritage the style of simple and user-friendly features of Scandinavia design, and its function and appearance are impeccable. But the using time of the Childers's products will be limit by the age of children. There are common problems in children products. Ordinarily, with the rapid changing of their physiology and psychology, the product life cycle was too shot to conserve resources, and this will cause a lot of waste. The proportion of the population was going down in the past few years in Developed country. Especially in China, onechild policy make some child products become the disposable products, because many products with complete functions were abandoned just for the child grown up.

How to prolong the lifespan of the products is the most effective way to reduce energy consumption.

With the increasing number of features, the increasing complexity of technical systems, the ideal degree may not necessarily improve if the technical system can eliminate some components while preserving the function of these components. This is consistent with reduce principles, the concept of green design certain. The baby carriage product technical system (we talked above) has reached an ideal state, but from the perspective of green design, the lifespan of the baby carriage was very limited, no more than 3 years. Through extensive research and observation, we found that many Childers's products that were in well function have been eliminated. Not because the product was bad, but because of a very simple reason, the children grow up, and their demands changed. Whatever the baby carriage designed perfect, children after the age of three years old, they never like to stay in the baby carriage, the demand has been terminated. Maybe the baby carriage can be given someone else, but the main problem existed in long-term idle baby products for children are corrosion.

With the improvement of living standards, Parents are more willing to buy new products for their children. Especially in China's, one-child policy make people would not like to use old children products for their only child.

Maybe the life-cycle of a baby carriage designed for 8 years, but most of them could not be used for so long time. Also an expensive car safety seats also have the same fate and this kind of products can cause huge waste.

\section{NEW DEMAND MODELING DESIGN BASED ON TRIZ INNOVATION THEORY}

TRIZ presents a systematic approach for analyzing the kind of challenging problems where inventiveness is needed and provides a range of strategies and tools for finding inventive solutions. One of the earliest findings of the massive research on which the theory is based is that the vast majority of problems that require inventive solutions typically reflect a need to overcome a dilemma or a trade-off between two contradictory elements.

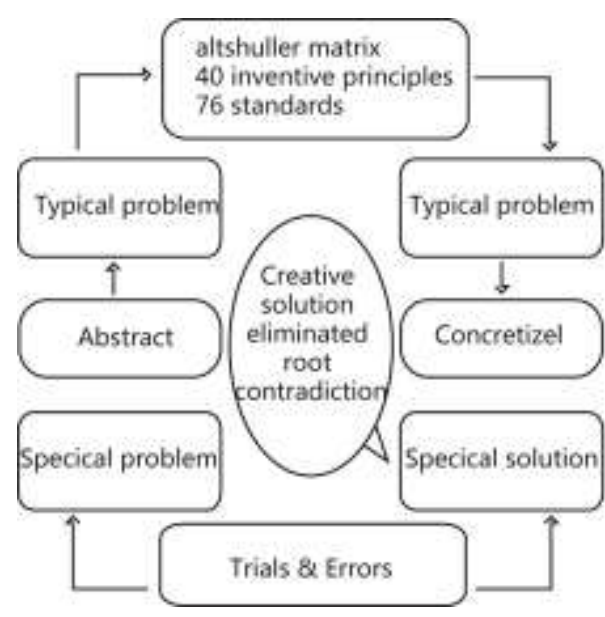

Figure2 TRIZ process for creative problem solving

One the fundamental method of green design was Reduce Principles which include three ways:

(1) Reduce the volume

(2)Reducing components

(3) Enhanced durability

Restricted by the Ergonomics that we could n't reduce the components limitless, and we wouldn't't reduction product's volume limitless. Good durability depends on the good martial which will increase the costs. The design principles of reduce also has limitations itself.

We can infer from Figure 2, there are several expanded functions radial from the core-function. We called "radial function", but these functions are concentrated in the same time period for the user to choose, but often ignore the user needs in the long term. The new challenge of green design concept is how to effectively reduce the consumption. Prolong the life cycle of products is one of the most direct and effective way to mend this shortcoming. A bottom-up approach to tackling the problem is that extends the products life-cycle and makes the function continually

The fundamental ways to reduce resources consumption is to reduce the number of products. It is required a very extensible design enabling virtually any form of extensions to the core function. It can meet the needs of users in a longer period of time.

For these reasons, article firstly analyzes the current problems in the development of products design based on the theory of Inventive Problem Solving o TRIZ is the Abbreviation of the Russian words; it means the theory of inventive problem solving,

TRIZ theory consider that any system is the ideal result to the evolutionary direction,Following this approach the ideal result eliminates all of the Harmful effects and make the useful effects flourish best. But when the system is more close to the ideal result, the cost is lower, and the efficiency is higher, utilization of the system is higher.

Based on the separation principle of TRIZ theory, we can further optimize the method of muti-functional design and make the needs continues: 
1. Time separation, considering the changing needs of user in different time; we should think about it and design it.

2. Divided the product function into modular, and the user can use every function independents.

3. Cultural background of users, the family values is different between the Confucian cultural circle in Asia and the western family culture. People, who living in a big family can share some products with their families, so considering all the needs of family members can improve the frequency of use of the product, and this may extend the products' life-cycle.

\section{DESIGN CASE}

Recently, however, there has been increased interest in the development of load-bearing materials and structures which have integral non-load-bearing functions, guided by recent discoveries about how multifunctional biological systems work. [3]

According to the function continuity theory, we redesign the baby carriage, bicycle, car safety seats; fitness twist car products such as functional continue to integrate experimental designs

1、 Bicycle for mother and children: using the Reverse Design Principle of TRIZ, put the children in the front for the mother to take care convenient, this bike Mothers can use this bike take their babies go shopping and do other activities in near distance, this product is low carbon safety and convenient. This function can be used in children 0-2 years of age, and then older children don't like to stay with the baby carriage.

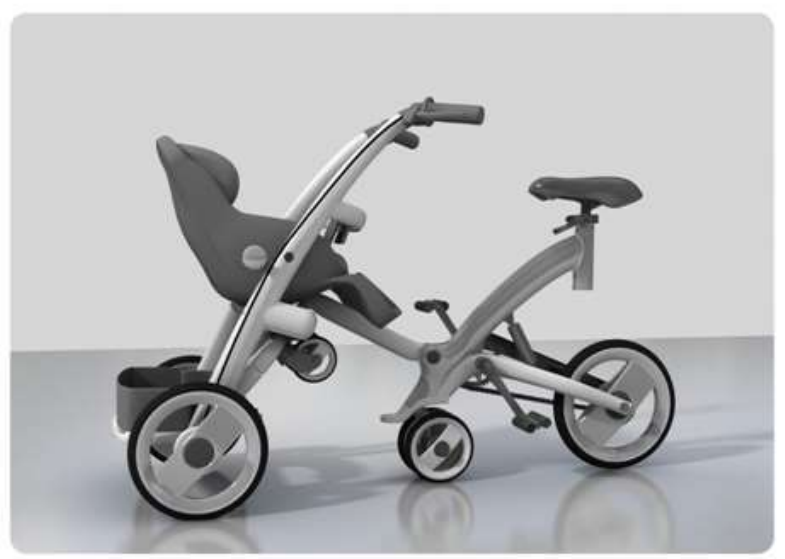

Figure3 Bicycle for mother and children

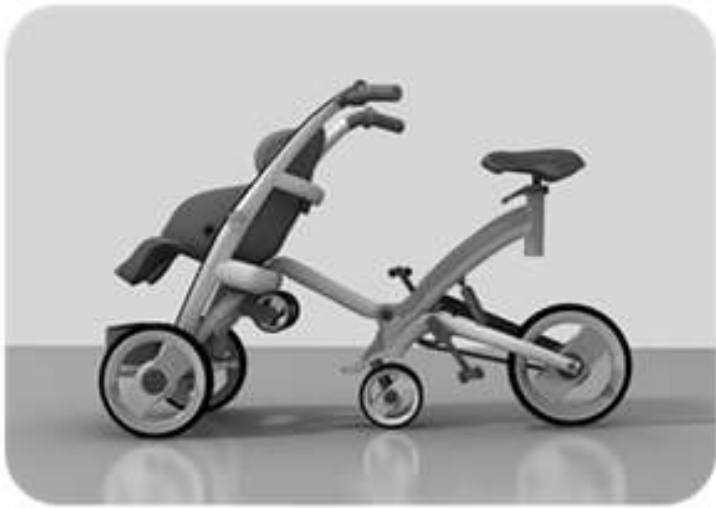

Figure4 Flexible installing modes of the seat

The product when not in use, how to effectively save storage space. We use the folding methods in design, to solve this problem, as shown in Figure5.

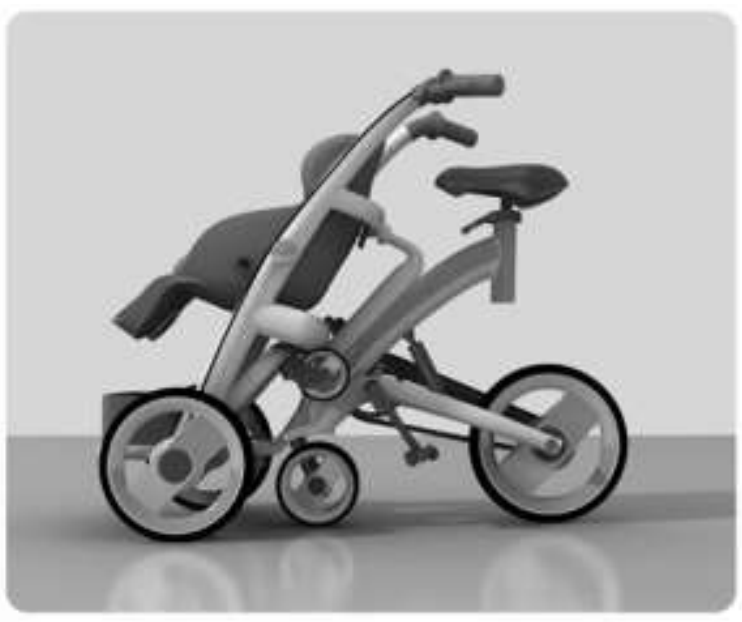

Figure5 the specific combination for space saving

2、Baby carriage: a baby carriage in the Bicycle for mother and children was independent and removable. It can be used continue to the children 0-5 age, as shown in Figure6.

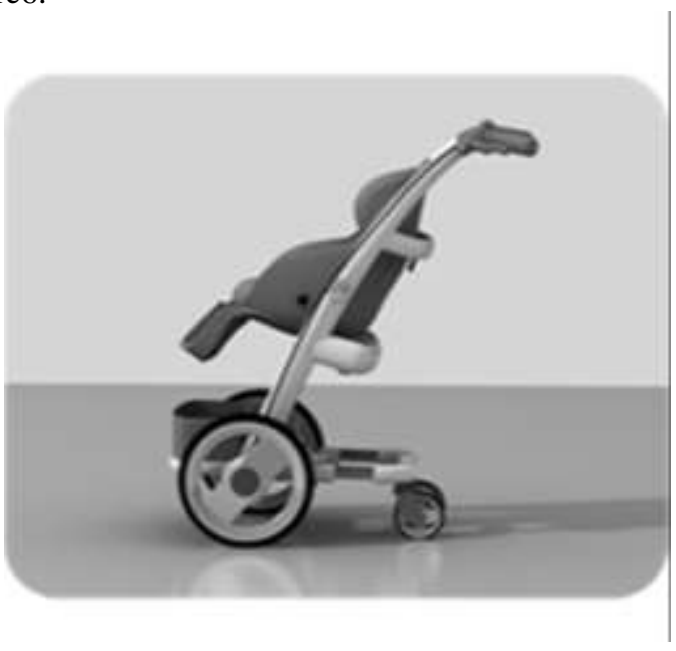

Figure6 baby carriage function 
3、 Car safety seat: A car safety seat in this baby carriage was independent and removable. Car safety seat can be used continue to the children 0-8 age, as shown in Figure7

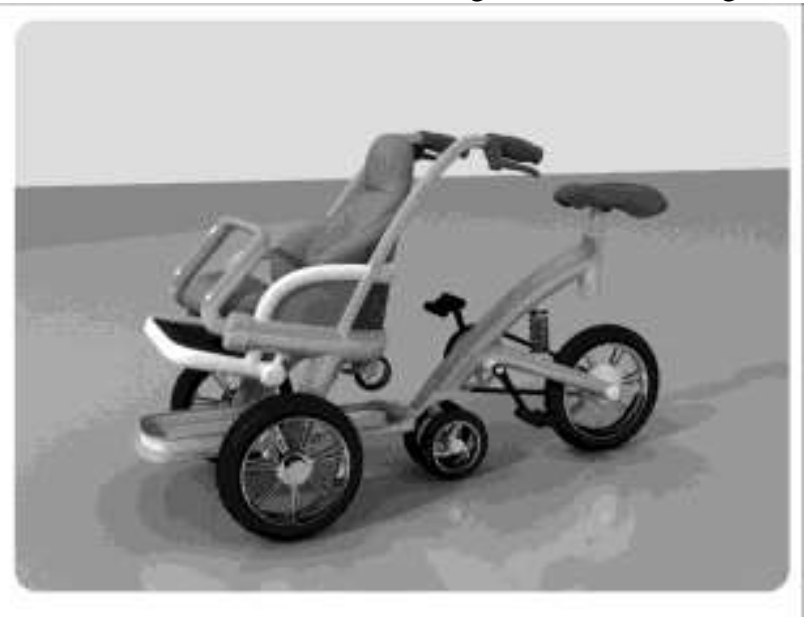

Figure7 the Car safety seat was independent and removable

4、 Shopping bicycle: When the children grow up, the bicycle function can be transformed to the shopping, and it can provide for the elder children to use, as shown in Figure8.

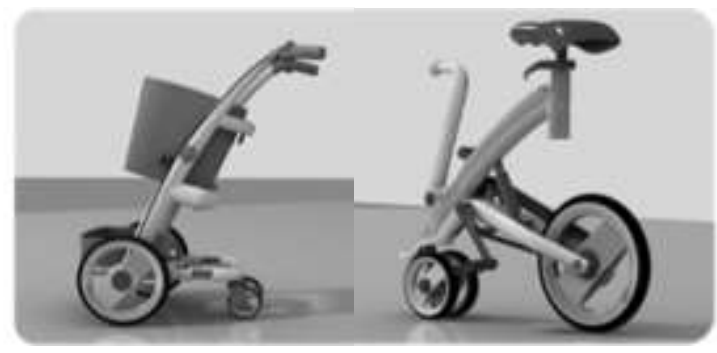

Figure8 Shopping bicycle

5、 Barrow: It can be used for shopping, carrying heavy loads.

6、 Fitness cars: This independently fitness entertainment is for adult and children over the age of 6 to use.

The product through the integration of various functions, the service life of the products can be extended to 10 years; the total resource consumption source is far lower than the purchase of different products alone. Thus the concept of the reduce

\section{CONCLUSION}

Based on the inventory analysis and impact assessment results, the following conclusions can be drawn concerning the contribution of the new demand modeling to prolong the product life cycle, which was one the reasons stated for carrying out this study:

- To remand the deficiencies of the existing multifunction design method, using the theory of TRIZ to find the solution.

- Re-understanding the relationship between the user needs and product functions, observe the Continuity of the product demand in a more wildly perspective, and using more micro perspective to analyze the product's functional modules to reach the ideal solution .

\section{REFERENCES}

[1] Kevin Perment, "Trade Promotion Management: An Excess of Excel!," AberdeenGroup blog, August 21, 2012.

[2] Reader's Guide to IDEF0 Function Models. Accessed 27 Nov 2008.

[3] A review of recent research on mechanics of multifunctional composite, Journal of Composite Structures 92 (2010) 2793-2810

[4] Niels Jungbluth. Feasibility of environmental product information based on life cycle thinking and recommendations for Switzerland. Journal of cleaner production. 2012, 28.

[5] Ruwen Qin ; D[5] Marco Garetti ; Paolo Rosa ; Sergio Terzi, Life Cycle Simulation for the design of Product-Service Systems. Computers in Industry, 2012, 63(4).

[6] avid A. Nembhard, Demand modeling of stochastic product diffusion over the life cycle. International journal of production economics EISCI, 2012, 137(2),

[7] Hui Cao ; Paul Folan, Product life cycle: the evolution of a paradigm and literature review from 1950-2009. Production Planning \& Control EISCI, 2012, 23(7/9).

[8] Li He-Cheng, Wang Yu-Ping, Product Configuration Design Oriented to Satisfaction for Diversity and Green Requirements. China Mechanical Engineering, 2012 23(7).

[9] YANG Bo;GAO Chang-qing;WANG Wei;LI Hai-tao,Pedigree Based Product Multi-Scheme Conceptual Design. Modular Machine Tool \& Automatic Manufacturing Technique, 2012, (3).

[10] The Choose and Application of Design Tools for Product Conceptual Design 。 MODERN MANUFACTURING TECHNOLOGY AND EQUIPMENT, 2008, (6)。 